\title{
ABORDAGEM ATUAL DA INSUFICIÊNCIA RENAL CRÔNICA CANINA
}

CRIVELENTI, Leandro Zuccolotto ${ }^{1}$

BORIN, Sofia ${ }^{2}$

BRUM, Alexandre Martini de ${ }^{3}$

\author{
Recebido em: 2009-03-31
}

Aprovado em: 2009-04-29

Issue DOI: $10.3738 / 1982.2278 .190$

RESUMO: A insuficiência renal crônica (IRC) é a consequência de uma gama de doenças renais, culminado na perda das funções excretora e concentradora dos rins. Considerada a principal afecção renal dos cães, destaca-se por sua progressão, e que independentemente da causa, leva à destruição lenta e irreversível dos néfrons. Muito se discute sobre terapias que retardem o avanço do quadro, porém a sobrevida dos animais insuficientes renais não depende somente da escolha da terapêutica medicamentosa ideal, mas da conscientização de proprietários e médicos veterinários em relação à importância do diagnóstico precoce, da identificação da doença renal desencadeadora do processo, da nutrição deste paciente e das manifestações clínico-laboratoriais apresentadas em suas diversas fases. Propõe-se com este artigo revisar as principais alterações decorrentes da IRC, enfocando pontos chaves da fisiopatologia, meios diagnósticos, terapêutica e manejo clínico do paciente renal crônico, visando ao retardamento da progressão da doença e propiciando maior qualidade de vida ao paciente.

Palavras-chave: Caninos. Rins. Insuficiência renal.

\section{CURRENT APPROACHING OF THE CHRONIC RENAL FAILURE}

SUMMARY: The chronic renal failure is a consequence of a lack of renal diseases, that culminates in loss of the kidney excretory and concentrator functions. It's considered the main renal dog affection and it's marked for its progression, in such a way that independently of the cause, leads to the gradual and irreversible destruction of the nefrons. Much discussion is made on therapies for delaying the disease advance, although the survival of animals with renal failure does not depend on only the choice of the ideal therapeutics but on the conscious of their owners and veterinarians in relation to the importance of the early diagnostic, identification of the original renal disease, the nutrition of this patient and clinic - laboratory aspects in its several phases. This article proposes to review the main changes caused by chronic renal failure, marking the principal points on physiopathology, diagnostic methods, therapeutic and clinic management of the chronic renal patient, in order to retard the disease progression and providing best life quality.

Keywords: Canines. Kidney. Renal failure.

1 Médico Veterinário graduado pela Universidade Federal de Uberlândia (UFU), Residente da Clínica e Cirurgia de Pequenos Animais na Universidade de Franca (UNIFRAN). Rua 7 de setembro, n. 360, Centro Batatais, SP. Cep. 14300-000. E-mail: crivelenti_lz@yahoo.com.br.

2 Médica Veterinária graduada pela Universidade Federal de Uberlândia (UFU), Residente da Clínica Médica de Pequenos Animais na Universidade Estadual Paulista (UNESP) - câmpus de Jaboticabal.

3 Médico Veterinário doutorando, Professor de Clínica Médica de Pequenos Animais na Universidade de Franca (UNIFRAN). 


\title{
ENFOQUE ACTUAL DE LA INSUFICIÊNCIA RENAL CRÓNICA
}

\begin{abstract}
RESUMEN: La insuficiencia renal crónica (IRC) es la consecuencia de una serie de enfermedades renales, culminando con la perda de las funciones de excreción e concentración de los riñones. Considerada la principal enfermedad renal de los perros, destacase por su progresión, y que, independientemente de la causa, conduce a la destrucción lenta e irreversible de los néfrons. Mucho debatese sobre las terapias que son capaces de reducir la progresión de la enfermedad, pero la supervivencia de los animales con insuficiencia renal no depende sólo de la elección de la terapia ideal, pero de la conciencia de los propietarios y veterinarios en relación a importancia del diagnóstico precoz, la identificación de la enfermedad renal desencadeadora del proceso, la nutrición este paciente e de las manifestaciones clínicas y laboratoriales en sus diversas etapas. Propone, con este artículo revisar las principales enmiendas derivadas de la IRC, centrándose en puntos clave de la fisiopatología, diagnóstico, tratamiento y conducción clínica, destinado al estancamiento de la progresión de la enfermedad y proporcionar una mejor calidad de vida para el paciente.
\end{abstract}

Palabras clave: Caninos. Riñones. Insuficiência.

\section{INTRODUÇÃO}

Os rins são essenciais à homeostase, não apenas por eliminarem produtos indesejáveis do metabolismo, como também por manterem constante o volume extracelular, a pressão osmótica e o equilíbrio eletrolítico. Como órgãos endócrinos, ativam a vitamina $\mathrm{D}_{3}$ (calcitriol), produzem eritropoetina, renina, prostaglandinas e cininas, além de serem sítios de atuação dos hormônios vasopressina, paratormônio (PTH) e aldosterona (POLZIN; OSBORNE, 1995).

A insuficiência renal crônica (IRC), principal afecção renal dos cães (FINCO, 1995; POLZIN; OSBORNE, 1995; SANCHES, 2005), reflete-se na perda da capacidade excretora e concentradora dos rins, com redução da filtração glomerular e consequente aumento nas concentrações plasmáticas de substâncias normalmente eliminadas, dentre as quais se destaca a ureia, componente do catabolismo protéico (POLZIN et al., 1997).

Do ponto de vista diagnóstico, tanto para fins clínicos quanto experimentais, a classificação do paciente com IRC sem os sinais clínicos de uremia é um desafio para os pesquisadores da área. Partindo do princípio de que todo insuficiente renal crônico apresenta uma doença renal crônica (DRC), a International Renal Interest Society (IRIS), determinou-se um estadiamento da IRC, padronizando-a em 4 estágios: 1 - ausência de azotemia, 2 - azotemia leve, 3 - azotemia moderada e 4 - azotemia severa (Tabela 1). Ainda, o subestadiamento destes pacientes é feito pela avaliação da relação proteína/creatinina urinárias (UP/C) e pela pressão arterial sistêmica conforme a Tabela 2. 
TABELA 1 - Estadiamento da insuficiência renal crônica segundo International Renal Interest Society

\begin{tabular}{ccc}
\hline ESTADIAMENTO & \multicolumn{2}{c}{ CREATININA (mg/dL) } \\
& CÃES & GATOS \\
\hline Estágio 1 & $<1,4$ & $<1,6$ \\
Estágio 2 & $1,4-2,0$ & $1,6-2,8$ \\
Estágio 3 & $2,1-5,0$ & $2,9-5,0$ \\
Estágio 4 & $>5,0$ & $>5,0$ \\
\hline
\end{tabular}

Adaptado de IRIS(2006)

TABELA 2 - Subestadiamento da insuficiência renal crônica canina segundo a International Renal Interest Society (IRIS), 2006.

\begin{tabular}{l|c|c}
\multicolumn{1}{c}{ SUBESTÁDIO } & \multicolumn{2}{c}{ VALOR DE UP/C } \\
\hline $\begin{array}{l}\text { Não proteinúrico (NP) } \\
\text { Proteinúria limítrofe (PL) }\end{array}$ & \multicolumn{2}{c}{$0,2-0,2$} \\
Proteinúrico (P) & \multicolumn{2}{c}{$>0,5$} \\
\hline & PRESSÃO ARTERIAL & PRESSÃO ARTERIAL \\
& SISTÓLICA (mmHg) & PIASTÓLICA (mmHg) \\
\hline Risco mínimo (N) & $150-150$ & $<95$ \\
Baixo risco (L) & $160-179$ & $95-99$ \\
Risco Moderado (M) & $=180$ & $100-119$ \\
Alto risco (H) & & $=120$ \\
\hline
\end{tabular}

O aumento dessas substâncias causam uma constelação de sinais clínicos conhecida como síndrome urêmica, que incluem o desequilíbrio hídrico e natrêmico, anemia, intolerância a carboidratos, distúrbios neurológicos, distúrbios gastrintéricos, osteodistrofias (FINCO, 1995), deficiência imunológica, acidose metabólica e processos inflamatórios que predispõem fibrose e esclerose renais (OTS et al., 2000).

Os distúrbios glomerulares primários são propostos como as principais causas de IRC em cães (GRAUER, 2005), porém amiloidose (RAVID et al., 1982), pielonefrite, urolitíase, displasia renal, nefrotoxinas, doença renal policística, hipercalcemia, linfoma, doenças autoimunes e imunemediadas e leptospirose (POLZIN et al., 1997; PRESSLER e VADEN, 2003) também são referidos na etiologia da doença.

A idade média do diagnóstico de IRC é de aproximadamente 7 anos, e a prevalência aumenta com o envelhecer dos cães (POLZIN et al., 1997). No entanto, a IRC congênita ou familiar, geralmente, acomete animais jovens na faixa etária de quatro meses a dois anos de idade (BITTENCOURT et al., 2004; CASTRO, 2005).

\section{FISIOPATOGENIA DA INSUFICIÊNCIA RENAL CRÔNICA}

Trata-se de uma doença progressiva que, independentemente da causa, leva à destruição lenta de uma porção dos néfrons, enquanto os néfrons ilesos remanescentes sofrem hipertrofia compensatória, por um determinado tempo. Porém, quando a IRC se instala, os néfrons hipertrofiados podem não conseguir mais manter a função renal (POLZIN; OSBORNE, 1995; 
WARE, 2006), mesmo quando retirada causa ou instituída terapêutica adequada (DOWN; KRAWIEC, 1996).

A diminuição do gradiente de concentração de sódio na medula renal pela redução dos nefróns funcionantes e, portanto, do número de alças de Henle, reduzem a capacidade de concentração da urina. A diminuição da hipertonicidade da medula renal reduz a pressão osmótica medular que atua na reabsorção passiva de água nos túbulos distais e nos ductos coletores, na presença de ADH (POLZIN et al., 1997). Assim, devido à poliúria e à polidipsia (PU/PD), o paciente renal crônico necessita de ter sempre água disponível (WARE, 2006).

Ressalta-se que consequente à poliúria ocorre depleção de vitaminas hidrossolúveis do complexo B, vitamina C e aumento da excreção de potássio (POLZIN; OSBORNE, 1995; WARE, 2006).

A nefropatia mais comum em cães é a de perda protéica (PRESSLER; VADEN, 2003), em que a proteinúria e a glomeruloesclerose podem ser devidas a hiperfiltração renal decorrente do aumento da função dos néfrons remanescentes (WARE, 2006). A proteinúria persistente, também, mostrou ser uma das causas da progressão da doença (OTS et al., 2000), pois induz um processo inflamatório constante devido ativação de citocinas inflamatórias no parênquima renal, causando fibrose túbulo-intersticial, havendo também evidências que as proteínas induzam apoptose das células tubulares (TRYGGVASON; PETTERSSON, 2003).

Dentre os fatores apontados como agravantes na progressão do quadro, destaca-se a ação do sistema renina-angiotensina-aldosterona (OTS et al., 2000), atribuindo-se à angiotensina II importante incremento na pressão sistêmica e glomerular, alto poder de fibrose e posterior glomeruloesclerose, que associada à proteinúria persistente, acarretam lesões túbulo-intersticiais, decorrentes da apoptose das células tubulares renais (TRYGGVASON; PETTERSSON, 2003; LEFEBVRE; TOUTAIN, 2004; LEES, 2004).

Os rins são susceptíveis à injúria hipertensiva, porém a arteríola aferente, como mecanismo de proteção renal, normalmente se contrai frente aumento da pressão arterial sistêmica. No entanto, em casos de IRC esse recurso é pouco responsivo, e toda mudança na pressão é transmitida diretamente para os rins (BURANAKARL et al., 2007). Ademais, é importante ressaltar que mesmo animais com a pressão arterial sistêmica dentro da normalidade podem apresentar hipertensão renal (LEFEBVRE; TOUTAIN, 2004).

A redução do metabolismo e o aumento das concentrações de gastrina e de hormônios paratireoideanos contribuem para o desenvolvimento de gastrite e hiperparatireoidismo secundário renal, respectivamente (WARE, 2006), sendo este último considerado um dos principais mecanismos envolvidos na progressão da IRC (CASTRO, 2005).

O aumento do paratormônio (PTH) advém da tentativa de manter a homeostase do cálcio (LAZARETTI et al., 2006). A diminuição da eliminação do fósforo, decorrente da redução da taxa de filtração, é o fator desencadeante do quadro, sendo a secreção exacerbada de PTH secundária ao aumento da concentração sérica deste eletrólito (FINCO, 1995; POLZIN et al., 1997). O fator de crescimento fibroblástico 23 (FGF 23), secretado em resposta à hiperfosfatemia, aumenta 
a excreção renal de fósforo e suprime a síntese de 1,25 calcitriol pelo rim, desenvolvendo papel importante na gênese do hiperparatireoidismo secundário renal (PERWAD et al., 2005). Em pacientes urêmicos com hiperparatireoidismo, as altas concentrações séricas de PTH estão associadas a baixas concentrações séricas de calcitriol, hipo ou normocalcemia e hiperfosfatemia, portanto, é difícil distinguir os efeitos indesejáveis do aumento do PTH, dos causados pela diminuição do calcitriol e cálcio e aumento do fósforo. (VANHOLDER et al., 2001).

$\mathrm{O}$ excesso de PTH diminui a atividade sérica da LDL, que resulta em prejuízo na remoção de lipídeos da circulação, ocasionando hiperlipidemia. Além desta, pode-se listar uma gama de alterações causadas pelo hiperparatireoidismo, tais como osteodistrofia, doenças cardiovasculares, alterações no sistema imune e anemia. A hiperfosfatemia e o aumento dos produtos cálcio-fósforo são os principais fatores envolvidos na calcificação vascular e mortalidade de pacientes com doença renal estágio final, além de induzir mineralização de outros tecidos moles, incluindo os próprios rins (AKMAL et al., 1990).

A dislipidemia, assim como nos humanos, representa o aumento do colesterol e triglicérides séricos, leva a formação de apolipoproteinas, que quando eliminadas pelos rins, causam danos semelhantes à proteinúria (DOWN; KRAWIEC, 1996).

Associado ao aumento da mortalidade e morbidade dos pacientes com IRC está o desenvolvimento de anemia (SANCHES, 2005), pois os rins são os principais sítios de produção da eritropoetina nos cães (MORITZ et al., 1997). Esta pode ser agravada pelas inúmeras lesões causadas pela uremia no trato gastrintestinal, levando a hematêmese, melena e hematoquesia (POLZIN; OSBORNE, 1995). Além disso, insuficiência renal pode modificar à proporção de fosfolípides e de colesterol na membrana dos eritrócitos, aumentando sua fragilidade e diminuindo seu tempo de vida (AKMAL et al., 1985; SÍLVIA et al., 2004). A produção excessiva do $\mathrm{PTH}$, também, contribui para o comprometimento da eritropoiese. Há evidências que esse hormônio iniba a proliferação de precursores eritróides na medula óssea. As poliaminas são toxinas urêmicas envolvidas na gênese da anemia. Estes compostos, entre eles a espermina, espermidina, putrescina e cadaverina, desempenham papel na proliferação e diferenciação celular e são conhecidos por se acumular no plasma de doentes com IRC. Acredita-se que atuam antagonizando a ação da eritropoetina. Outras toxinas urêmicas que tem papel importante no desenvolvimento da anemia são as citocinas pró inflamatórias, como a interleucinas-1, fator de necrose tumoral- $\alpha$ e o interferon- $\alpha$, que causam efeitos supressores da eritropoiese (MACDOUGALL, 2001).

Além disso, deficiência de ferro foi verificada em $57 \%$ dos pacientes pré-dialíticos humanos. Tal fenômeno pode ser explicado pela anorexia ou pelo aumento do peptídeo hepicidina, que inibe a absorção intestinal de ferro (CANZIANI et al., 2006). 


\section{DIAGNÓSTICO}

Estratégias efetivas, para o diagnóstico precoce da IRC, devem ser baseadas na detecção de alterações mínimas na função renal (LEES, 2004).

Ressalta-se que a creatinina e a ureia séricas, geralmente, encontram-se aumentadas nos pacientes com sinais clínicos (FINCO, 1995), porém pacientes assintomáticos (estágio 1) podem, em alguns momentos, apresentá-las dentro dos valores normais, ou até mesmo, por fatores extra-renais como pela redução de massa muscular ou alterações no fluido corpóreo (LEES, 2004).

A dosagem sérica de Cistina C não sofre influências do meio externo, pois é constantemente produzida por todas as células nucleadas do organismo e filtradas pelo glomérulo (ANTOGNONI et al., 2005), podendo essa ser usada nos casos em que a creatinina apresente-se dentro ou próxima dos limites de normalidade. No entanto, neoplasias como os melanomas devem ser investigados, pois tendem a aumentar sua concentração sérica (BRAUN et al., 2002).

Dentre os meios diagnósticos, a depuração renal de creatinina avalia a taxa de filtração glomerular, refletindo com exatidão a função renal. Pode ser calculada, usando-se tanto a creatinina endógena (cleareance de creatinina) quanto a exógena. A técnica mais utilizada realiza a mensuração do cleareance de creatinina, a qual requer, além de amostra sanguínea, a utilização de gaiolas metabólicas para obtenção de urina acumulada em 24 horas, podendo ser realizada em animais azotêmicos sem contra-indicações (POLZIN et al., 1997).

A proteinúria é rotineiramente mensurada por fitas reagentes, contudo essa metodologia pode apresentar erros. Sendo assim, a quantificação da proteína excretada em 24 horas ou o cálculo do UP/C (relação entre a proteína e a creatinina urinárias) são métodos mais fidedignos, servindo de parâmetros indicativos da progressão da doença (TRYGGVASON; PETTERSSON, 2003; GRAUER, 2005; BURANAKARL et al., 2007). Valores de UPC superiores a 0,5 podem indicar o início de lesão renal ou estadiamento 1 (LEES, 2004).

A excreção fracionada consiste na depuração de vários solutos na urina comparando-se a de creatinina (FINCO, 1995). Na IRC, alguns solutos podem ter sua excreção aumentada, como é o caso do magnésio, decorrente do hiperparatireoidismo renal ou do dano tubular. Já, as excreções de fósforo e de ferro podem estar diminuídas, indicando precocemente a redução da taxa de filtração glomerular (BURANAKARL et al., 2007).

A acidose metabólica é também incriminada na progressão da IRC, porém muitas vezes só é evidenciada, quando já existe um dano renal muito grave, sendo muitas vezes difícil e onerosa sua quantificação (ELLIOTT et al., 2003).

A radiografia simples pode confirmar a presença de rins pequenos. Na maioria dos casos, a ultrassonografia renal mostrará córtices renais difusamente ecodensos, com perdas dos limites corticomedulares (POLZIN et al., 1997). Cistos podem ser encontrados acidentalmente, porém, geralmente, não estão associados à disfunção renal (GROOTERS, et al. 1997). Durante a necropsia, podem ser observados cistos, neoplasias, abscessos, granulomas e hematomas 
renais (POLZIN et al., 1997; CUYPERS, et al. 1997). Abscessos são incomuns e podem estar associados a neoplasias e abscessos em órgãos como fígado e baço.

A citologia por agulha fina é uma técnica invasiva indicada em casos de processos infiltrativos, inflamatórios e neoplásicos, devendo ser realizada com cautela após os exames de rotina (BORJESSON, 2003).

$\mathrm{Na}$ diferenciação das glomerulopatias, o diagnóstico definitivo é realizado pela histopatologia do córtex renal(WARE, 2006), a qual é indicada em casos de proteinúria persistente, onde já foram realizados os exames de rotina. $\mathrm{O}$ fragmento pode ser coletado percutaneamente usando a técnica keyhole laparoscópica ou guiada por ultrassom. Frequentemente, a laparotomia é a melhor escolha para biopsia renal, por possibilitar a visualização dos rins, adequado tratamento de hemorragias e coleta de fragmentos mais representativos (GRAUER, 2005).

\section{TRATAMENTO}

O objetivo do tratamento de pacientes com IRC é o de retardar a progressão da doença (LEFEBVRE; TOUTAIN, 2004). Dessa forma, a fluidoterapia parenteral é a conduta terapêutica mais importante para restaurar o volume extracelular, corrigir o equilíbrio eletrolítico e reduzir a azotemia no paciente que apresenta-se em crise urêmica com sinais clínicos e alterações laboratoriais (POLZIN et al., 1997).

O suporte nutricional há anos vem sendo estudado em medicina humana e atualmente tem sido considerado como ponto chave no controle da insuficiência renal canina (REFSAL et al., 2001; VEADO et al., 2002). Dietas com teores reduzidos de proteína, fósforo, sódio e a suplementação de ácidos graxos $\Omega 3$ podem ser benéficos (POLZIN et al., 1997). No caso de vômitos crônicos, pode-se optar pelo uso da nutrição parenteral, que consiste na administração de todos ou parte das exigências nutricionais diárias através da via venosa (HALL, 1996).

O uso de cetoanálogos (ß-cetoácidos de aminoácidos) tem sido proposto nos protocolos de tratamento das insuficiências renais em cães. Esses compostos, ao mesmo tempo em que captam o nitrogênio da circulação, são transformados em aminoácidos essenciais. Assim, os cetoanálogos servem de complemento nutricional ao fornecerem aminoácidos de alto valor biológico, permitindo que as dietas possam conter menor teor de proteínas, reduzindo assim a concentração de ureia sérica (VEADO et al., 2002).

O tratamento da hipertensão arterial deve ser instituído ato contínuo ao seu diagnóstico, e mantido ad eternum com ajustes periódicos. Nesses casos, sugere-se a utilização dos inibidores da enzima conversora de angiotensina (iECA) como o enalapril e o benzepril (ambos nas doses de 0.25 - $0.5 \mathrm{mg} / \mathrm{kg}$ a cada 12 horas, pela via oral) (TRYGGVASON; PETTERSSON, 2003). Como medida emergencial, pode-se optar por infusão contínua de nitroprussiato de sódio, hidralazina ou selo de nitroglicerina como forma de redução da pressão rapidamente (LEFEBVRE; TOUTAIN, 2004).

Nucleus Animalium, v.1, n.1, maio 2009 
Os iECAS, também, podem ser usados para diminuição da proteinúria mesmo em animais que não são hipertensos (LEFEBVRE; TOUTAIN, 2004), pois também agem como renoprotetores (OTS et al., 2000; GRAUER, 2005). Estudos em ratos mostraram bons resultados com o uso de losartan, irbersartan, ramipril, benazepril e captopril, chegando a diminuir em até $97 \%$ a proteinúria e aumentando a sobrevida dos animais tratados (TRYGGVASON; PETTERSSON, 2003). O enalapril foi efetivo na redução da proteinúria na dose de $0,5 \mathrm{mg} / \mathrm{kg}$ BID em cães (GRAUER, 2005).

Em relação às complicações da $\mathrm{IRC}$, o tratamento do hiperparatireoidismo secundário renal, síndrome de hipercoagulabilidade, dislipidemia, anemia, acidose metabólica, hipocalemia e gastropatia urêmica são de extrema importância.

$\mathrm{Na}$ tentativa de minimizar a evolução do hiperparatireoidismo renal secundário, devese utilizar dieta para nefropatas restrita em fósforo e optar pelo uso de quelantes de fósforo intestinais como o hidróxido de alumínio (30-90 mg/kg, VO BID) junto com a alimentação (CASTRO, 2005). Compostos dopaminérgicos, como a dopamina, apresentam ação fosfatúrica. Apesar da infusão de dopamina, na dose de $1 \mu \mathrm{g} / \mathrm{kg} / \mathrm{min}$, ter sido capaz de reduzir a concentração sérica de fósforo em cães nefropatas (BRUM, 2007), estudos ulteriores são necessários para avaliar sua segurança e real efeito nesses casos.

Em humanos, recomenda-se o tratamento com calcitriol, quando os níveis séricos de PTHi e 25OHD encontram-se acima dos limites de referência, verificando-se que a suplementação com ergocalciferol foi capaz de reduzir os níveis de PTH. Porém efeitos adversos do tratamento como maior absorção intestinal de Ca e $\mathrm{P}$ podem produzir hipercalcemia e hiperfosfatemia. Por tais motivos, os níveis séricos de PTHi, Ca e P devem ser monitorados durante o tratamento com calcitriol, e a dose, ajustada para manter seus níveis recomendados. Nos casos em que os níveis de P e PTHi sérico permaneçam elevados, deve-se considerar a indicação de paratireoidectomia (KAHROL; JORGETTI, 2008).

Em casos de glomerulonefrite, podem-se utilizar drogas antiplaquetárias, incluindo inibidores da troboxano sintetase. O ácido acetilsalicílico $(0,5$ a $5 \mathrm{mg} / \mathrm{Kg}$, VO, SID-BID para cães), atenuando as alterações histológicas e funcionais associadas a glomerulonefrite por imunocomplexos (LONGHOFER et al., 1991), diminui as chances de tromboembolismo.

A lovastatina pode ser usada na dose de 10 a $20 \mathrm{mg}$ PO SID na hipercolesterolemia em cães com síndrome nefrótica que não respondam somente com a dieta (WARE, 2006).

Com relação à anemia na IRC, pode-se utilizar eritropoetina recombinante humana (rhEPO), porém seu uso deve ser criterioso, visto que em 20 a 40\% dos casos o paciente poderá produzir anticorpos contra este hormônio, podendo levar a anemia grave dependente de transfusão (CASTRO, 2005). Desse modo, benefícios podem ser alcançados com essa terapêutica em animais com anemia não regenerativa pela hipoplasia de medula óssea (RANDOLPH et al., 2004). A terapia deve ser associada à administração de ferro, pelo aumento de sua demanda durante a eritropoiese induzida pela rhEPO (ASSARASAKORN et al., 2008). A deficiência de ferro pode ser caracterizada pela presença de porcentagem de saturação menor que $20 \%$ 
associada ou não a ferritina menor que $100 \mathrm{ng} / \mathrm{mL}$, e sua correção pode ser realizada pela via intravascular (CANZIANI et al., 2006).

$\mathrm{O}$ uso de bloqueadores de receptores $\mathrm{H}_{2}$, como a ranitidina e a famotidina devem ser usados em pacientes com gastrite induzida pela uremia. Em casos de úlceras gastrintestinais, pode-se optar pelo sucralfato e omeprazol (POLZIN; OSBORNE, 1995).

As doses de drogas, com eliminação renal, devem ser reduzidas, ou o seu intervalo aumentado (WARE, 2006), evitando sobrecarga da função renal já comprometida.

A hemodiálise consiste em uma terapia de substituição temporária da função renal, extracorpórea, indicada para controlar as desordens causadas pela uremia, tais como anúria, hiper-hidratação, desequilíbrio hidreletrolítico e ácido-base e intensa azotemia (WARE, 2006). Porém, é uma terapia complexa e pode apresentar efeitos adversos que são difíceis de serem distinguidos dos sintomas de uremia em si. Esses efeitos constituem a síndrome do equilíbrio da diálise, principalmente, em animais de baixo peso, que apresentam hipotensão, ativação de mediadores inflamatórios e aumento de estresse oxidativo (COWGILL; FRANCEY, 2006).

$\mathrm{Na}$ medicina humana, pacientes terminais com IRC se tornam progressivamente oligúricos/anúricos e são submetidos a intervenções dialíticas (hemodiálise ou diálise peritoneal) ou transplante renal. Já na medicina veterinária, a hemodiálise é muitas vezes realizada em animais ainda poliúricos, os quais poderiam se beneficiar do tratamento convencional e da fluidoterapia.

\section{CONSIDERAÇÕES FINAIS}

O diagnóstico precoce da insuficiência renal crônica é indiscutível, devendo-se retirar as causas base e promover o manejo adequado do paciente, visando ao retardamento da progressão da doença e maior qualidade de vida para o paciente.

A sobrevida de animais insuficientes renais depende muito da conscientização dos proprietários; tanto em relação à alimentação, quanto à administração das medicações corretamente. Deve-se sempre salientar de que se trata de uma doença progressiva e incurável, que apresenta somente controle clínico.

Devido às diferentes sintomatologias clínicas do paciente renal canino e humano, ressaltase que a opção pelo tratamento dialítico deve ser considerada nos casos de insuficiência renal crônica agudizada, quando os pacientes não respondam a terapia medicamentosa convencional ou estejam anúricos. 


\section{REFERÊNCIAS}

AKMAL, M. et al. Excess parathyroid hormone adversely affects lipid metabolism in chronic renal failure. Kidney International, New York, v. 37, p. 854-858, 1990.

ANTOGNONI, M.T. et al. Use of serum cistatin C determination as a marker of renal function in the dog. Veterinary Research Communications, Amsterdam, v. 29, n.2, p. 265-267, 2005.

ASSARASAKORN, S.; KAEWTHAMASORN, M.; NIWETPATHOMWAT, A. A retrospective study of clinical use of recombinant human erythropoietin for treatment of anemia in dogs with renal failure in Bangkok, Thailand. Comparative Clinical Pathology, London, v.17 p. 165$170,2008$.

BITTENCOURT, E. et al. Nefropatia juvenil progressiva associada à displasia renal em Lhasa apso: relato de caso. Clínica Veterinária, São Paulo, n. 48, p. 24-26, 2004.

BORJESSON, L. B. Renal Cytology. The Veterinary clinics of North America. Small animal practice, Philadelphia, n. 33, p. 119-134, 2003.

BRAUN, J. P.,et al. Plasma Cystatin C in the dog: reference values and variations with renal failure. Comparative Clinical Pathology, London, v. 11 p. 44-49, 2002.

BRUM, A. M. Avaliação da função renal de cães sadios e nefropatas sob infusão de dopamina. Dissertação (Mestrado em Clinica Médica Veterinária). Faculdade de Medicina, Campus de Jaboticabal, Universidade Estadual Paulista "Julio de Mesquita Filho". Jaboticabal, 2007.

BURANAKARL, C. et al. Relationships Between Degree of Azotaemia and Blood Pressure, Urinary Protein:Creatinine Ratio and Fractional Excretion of Electrolytes in Dogs with Renal Azotaemia. Veterinary Research Communications, Amsterdam, v. 31, p. 245-257, 2007.

CANZIANI, M. E. F. et al. Deficiência de ferro e anemia na doença renal crônica. Jornal Brasileiro de Nefrologia, São Paulo, v. 28, n. 2, p. 86-90, 2006.

CASTRO, M. C. N. Prolongando a vida do paciente com insuficiência renal crônica. Clínica Veterinária, São Paulo, n. 58, p. 50-58, 2005.

COWGILL, L. D.; FRANCEY, T. Hemodialisys. In: DiBARTOLA, S. P. Fluid, electrolyte, and acid-base disorders in small animal practice. 3 ed. Saunders Elsevier: Philadelphia. 2005. 702p.

Nucleus Animalium, v.1, n.1, maio 2009 
DOWN, L. K.; KRAWIEC, D. R. Dylipoproteinemia of chronic renal failure: it's relevance to canine progressive canine disease. Compendium of Continuing Education for the Practicing Veterinarian (Small Animal). Philadelphia: Elsevier Health Science, v. 18, n. 1, 1996.

ELLIOTT, J.; SYME, H. M.; MARKWELL, P. J. Acid-base balance of cats with chronic renal failure: effect of deterioration in renal function. Journal of Small Animal Practice, Oxford, v. 44, p. 261-268, 2003.

FINCO, D. R. Evaluation of renal functions. In: Osborne, C. A.; FINCO, D. R. Canine and Feline Nephrology and Urology. Willians e Wilkins: Baltimore. 1995, p. 29-46.

GRAUER, G. F. Canine glomerulonephritis: new thoughts on proteinuria e treatment. Journal of Small Animal Pratice, Oxford, v. 46, p. 469-478, 2005.

GROOTERS, A. M. et al. Renomegaly in Dogs and Cats. Part II. Differential Diagnoses. Compendium of Continuing Education for the Practicing Veterinarian (Small Animal). Philadelphia: Elsevier Health Science, v. 19, n. 9, p. 1213-1229, 1997.

HALL, E. Gastrintestinal problems. In: KELLY, N. C., WILL, J. M. Manual of Companion Animal Nutritional and Feeding. Ames: Iowa State University Press, 1996.

KAHROL, C.; JORGETTI, V. Prevenção e Tratamento do Hiperparatireidismo Secundário na DRC. Jornal Brasileiro de Nefrologia, São Paulo, v. 30, p. 9-14, 2008.

LAZARETTI, P. et al. Concentração sérica de paratormônio intacto em cães com insuficiência renal crônica. Arquivo Brasileiro de Medicina Veterinária e Zootecnia, Belo Horizonte, v.58, n.4, p.489-494, 2006.

LEES, G. E. Early diagnosis of renal disease and renal failure. The Veterinary clinics of North America. Small animal practice, Philadelphia, v. 34, p.867, 2004.

LEFEBVRE, H. P.; TOUTAIN, P. L. Angiotensin-converting enzyme inhibitors in the therapy of renal diseases. Journal of veterinary pharmacology and therapeutics, Oxford, v. 27, p. 265-281, 2004.

LONGHOFER, S. L. et al. Effects of thromboxane synthetase inhibition on immune complex glomerulonephritis. American Journal of Veterinary Research, Chicago, v. 52, n.3, 480487,1991 . 
MACDOUGALL, I. C. Role of uremic toxins in exarcerbating anemia in renal failure. Kidney international, New York, v. 59, n. 78, p. 67-72, 2001.

MORITZ, K. M.; LIM, G. B.; WINTOUR, E. M. Developmental regulation of erythropoietin and erythropoiesis, American journal of physiology. Regulatory, integrative and comparative physiology, Bethesda, v. 273, p. 1829-1844, 1997.

OTS, M.; PECHTER, U.; TAMM, A. Characteristics of progressive renal disease. Clinica chimica acta; international journal of clinical chemistry, Amsterdam. v. 297, p. 29-41, 2000 .

PERWAD, F. et al. Dietary and serum phosphorus regulate fibroblast growth factor 23 expression and 1,25-dihydroxyvitamin D metabolism in mice. Endocrinology, Springfield, v. 146, p. 53585364, 2005.

POLZIN, D. J.; OSBORNE, C. A. Pathophysiology of renal failure and uremia. In: OSBORNE, C. A.; FINCO, D. R. Canine and feline nephrology and urology, Williams and Wilkins: Baltimore. 1995. p. 335-367.

POLZIN, D. J. et al. Insuficiencia renal crônica In: ETTINGER, S. J.; FELDMAN, E. C. Tratado de medicina interna veterinária, 4 ed. São Paulo: Manole, 1997. p. 2394-2431.

PRESSLER, B. M.; VADEN, S. L. Managing renal amyloidosis in dogs and cats. Veterinary Medicine, Kansas City, v. 98, n. 4, p.320-333, 2003.

RANDOLPH, J. F. et al. Clinical efficacy and safety of recombinant canine erythropoietin in dogs with anemia of chronic renal failure and dogs with recombinant human erythropoietininduced red cell aplasia. Journal of the veterinary Internal Medicine, Philadelphia, v. 18, n. 1, p. 81-89, 2004.

RAVID, M. et al. Prolonged dimethylsulphoxide treatment in 13 patients with systemic amyloidosis. Annals of the Rheumatic Diseases, London. v. 41, p. 587-592, 1982.

REFSAL, K. R. et al. Update on the diagnosis and treatment of disorders of calcium regulation. The Veterinary clinics of North America. Small animal, Philadelphia, v. 31, n. 5, p.1043$1062,2001$.

SANCHES, O. C. Histopatologia da série eritróideda medula óssea de cães com insuficiência renal crônica. 2005. Tese (Mestrado em Medicina Veterinária) - Faculdade de Medicina Veterinária e Zootecnia. Universidade Estadual Paulista “Julio de Mesquita Filho". Botucatu. 
SÍLVIA, F. E. et al. S. Fragilidade osmótica eritrocitária em gatos acometidos por hepatopatias e gatos com insuficiência renal. Ciência Rural, Santa Maria, v. 34, n. 2, p. 413-418, 2004.

Staging System for Chronic Kidney Disease (CKD). In: IRIS - International Renal Interest Society, 2006. Disponível em <Http:// www.iris-kidney.com>. Acessado em 08 mar 2009.

TRYGGVASON, K.; PETTERSSON, E. Causes and consequences of proteinuria: the kidney filtration barrier and progressive renal failure. Journal of Internal Medicine, v. 253, p. 216$224,2003$.

VANHOLDER, R. et al. Uremic toxicity: present state of the art. The International journal of artificial organs, Milano, v. 24, n. 10, p. 695-725, 2001.

VEADO, J.C.C. et al. Uso de cetoanálogo na terapia da insuficiência renal canina. Arquivo Brasileiro de Medicina Veterinária e Zootecnia, Belo Horizonte, v. 54, n. 5, p. 543-545 2002.

WARE, W. A. Glomerulopatias. In: NELSON, R. W.; COUTO, C. G. Medicina Interna de Pequenos Animais. 3 ed. Guanabara Koogan: Rio de Janeiro, 2006. p. 575-582. 